\title{
Searching for Survivor-Specific Services at NCI-Designated Comprehensive Cancer Centers: A Qualitative Assessment
}

\author{
Betsy Rolland, PhD, MLIS, MPH, ${ }^{a}$ and Jordan Eschler, $\mathrm{PhD}, \mathrm{MS}^{\mathrm{b}, \mathrm{c}}$
}

\begin{abstract}
Background: Cancer survivorship presents unique medical, psychosocial, and logistical challenges for survivors, their caregivers, and clinicians. $\mathrm{NCl}$-designated Comprehensive Cancer Centers (CCCs) serve a unique role in the delivery of cancer care because they not only provide care but also serve as a model for community oncologists and clinics through the development and dissemination of standards of care. Survivors, their caregivers, and clinicians look to CCCs to provide information about how to navigate the transition from active to posttreatment care. However, there is wide variability in the types of resources CCCs make available on their websites. The goal of our assessment was to understand the types of posttreatment survivor-specific resources CCCs are providing on their website. Methods: We analyzed the websites of the 47 CCCs to evaluate survivor-specific resources around the 4 components of survivorship care described by the Institute of Medicine guidelines for survivorship care plans: surveillance, prevention, intervention, and coordination. Results: 0 f the 47 CCCs, $74.5 \%$ $(n=35)$ had discoverable survivor-specific services on their websites. Despite our inclusive approach to coding, few websites contained extensive information targeted at survivors, their caregivers, or clinicians. Only the coordination and intervention elements were discussed by at least half of the CCCs. From the vantage point of cancer survivors, their lay caregivers, loved ones, or clinicians, there is limited information about survivor-specific services on the websites of the 47 CCCs. This dearth of information translates into substantial work for these groups to find the resources they may need. Conclusions: The CCCs have an opportunity to serve as information hubs and to lessen the amount of work associated with survivorship. As models of cancer care delivery, the CCCs can also set the standard for community oncologists and clinics for delivery of care that improves the quality of life for survivors.
\end{abstract}

J Nat/ Compr Canc Netw 2018;16(7):839-844 doi: 10.6004/jnccn.2018.7019

Improvements in early detection, treatment, and follow-up, combined with an aging population, have led to an increase in the number of people surviving cancer. ${ }^{1}$ Recently, Bluethmann et $\mathrm{al}^{2}$ projected that there will be 26.1 million US cancer survivors by 2040. Survivorship carries with it unique medical, psychosocial, and logistical challenges, which may include health problems arising from the treatments themselves, feelings of loss and anxiety about the future, or the financial impact of expensive cancer treatments., ${ }^{1,3}$ As the population of cancer survivors grows, clinicians are grappling with

anniversity of Wisconsin-Madison Carbone Cancer Center, Madison, Wisconsin; 'Information School, University of Washington, Seattle, Washington; and 'Center for Communication and Health, Northwestern University, Evanston, Illinois.

Submitted November 13, 2017; accepted for publication February 22, 2018.

The authors have disclosed that they have no financial interests, arrangements, affiliations, or commercial interests with the how best to meet these needs, and survivors are looking for information about how to transition from active to posttreatment care.

In response to increasing survivorship, NCI-designated Comprehensive Cancer Centers (CCCs) will be called on to take the lead for community providers and to act as sources of information for survivors, their caregivers, and clinicians as they navigate the next phase of care. Designation as a CCC requires evidence of the provision of exceptional care and conduct of translational research activities in the basic, clinical, and population manufacturers of any products discussed in this article or their competitors.

Author contributions: Study concept and design: All authors. Data acquisition: All authors. Data analysis and interpretation: All authors. Manuscript preparation: All authors. Critical revision: All authors.

Correspondence: Betsy Rolland, PhD, MLIS, MPH, University of WisconsinMadison Carbone Cancer Center, 800 University Bay Drive, 210-19, Madison, WI 53705. Email: brolland@wisc.edu 
Rolland and Eschler

sciences, with a special emphasis on ensuring that discoveries have a positive impact on the CCC's catchment area. CCCs occupy a unique space in the field of cancer care delivery because they provide care to patients while also engaging in cutting-edge research and, through organizations such as NCCN, developing and disseminating guidelines around standards of care. Although only a small percentage of survivors will receive their care at $\mathrm{CCCs},{ }^{3}$ community oncologists and clinics look to CCCs for such guidance.

Recent work by Leppin et al, ${ }^{4}$ Eschler et al, ${ }^{5}$ and Klasnja et $\mathrm{al}^{6}$ have described the enormous burden placed on patients and their caregivers during medical treatment, including finding information that they need to deal with their condition. CCCs are in a position to impact the lives of cancer survivors by minimizing the amount of information-seeking work patients and their loved ones must perform. Much of this information-seeking occurs online. According to the Pew Research Center's Internet \& American Life Project, $72 \%$ of surveyed internet users have searched for health information online in the past year. ${ }^{7}$ Furthermore, in a review of the Health Information $\mathrm{Na}$ tional Trends Survey (HINTS) data, Finney Rutton et $\mathrm{al}^{8}$ found that survivors searched for cancer information more frequently than those with a family history of cancer and those with no history of it, with $44 \%$ starting with their providers and 36\% starting with the internet.

Because of their position as leaders in the delivery of cancer care, CCCs will be expected to provide accessible, usable, comprehensive online resources for survivors and their caregivers. But are they doing so? To answer this question, we evaluated the information resources available to survivors by assessing, from the point of view of survivors and/or caregivers, what types of resources could be found on the CCC websites to help survivors manage their posttreatment lives.

\section{Methods}

The goal of our assessment was to understand the types of posttreatment, survivor-specific resources CCCs are providing on their website. We did not devise a new set of survivorship services to assess information available. Instead, we analyzed the sites specifically for the 4 components of survivorship care described by the Institute of Medicine (IOM) guide- lines for survivorship care plans (SCPs). The components of survivorship care are also used in Table $1^{3}$ :

- Surveillance for new or recurrent cancer (ongoing screening)

- Prevention of new or recurrent cancer (nutrition, smoking cessation, tanning/sunscreen use, exercise, genetic screening)

- Intervention for secondary cancers or late-term effects of treatment (mental health, pain control, rehabilitation, dermatology, fertility, alternative/ complementary medicine)

- Coordination plans between oncology care teams and primary care physicians (SCPs)

We also wanted to understand how the CCCs characterized survivorship programs and their services, including available information about the program leadership and staffing.

Two coders (B.R., J.E.) assessed the CCC websites on the availability of survivor-specific information in each of these 4 IOM components, as well as the details of the survivorship program. CCCs received a "1" if we identified survivor-specific information or a " 0 " if no information could be identified in that category. It was not enough to have smoking cessation services at the CCC; rather, a CCC needed to have a smoking cessation program specifically targeted at posttreatment survivors or survivor-specific smoking cessation information. We sought to err on the side of inclusion and allowed for a very broad definition of both the resources themselves and the resources' focus on survivors.

To find these resources, we began by using a search engine, as do $77 \%$ of online health information seekers. ${ }^{7}$ As of February 2017 when we began our analysis, there were 47 CCCs according to NCI's Office of Cancer Centers (see supplemental eAppendix 1 , available with this article at JNCCN.org). On the Google homepage, we entered the search term "[CCC full name] survivorship" for each of the CCCs to identify those that had any survivorship information on their websites. If the search returned no results, we tried "[CCC full name] survivor." These 2 search terms yielded the same results; if the first search term failed to return any relevant results, so did the second. We assessed only the 35 CCC sites that returned Google search results; although we browsed the 12 sites for which no search results were returned (given that browsing is a variant of finding relevant infor- 
Survivor-Specific Services

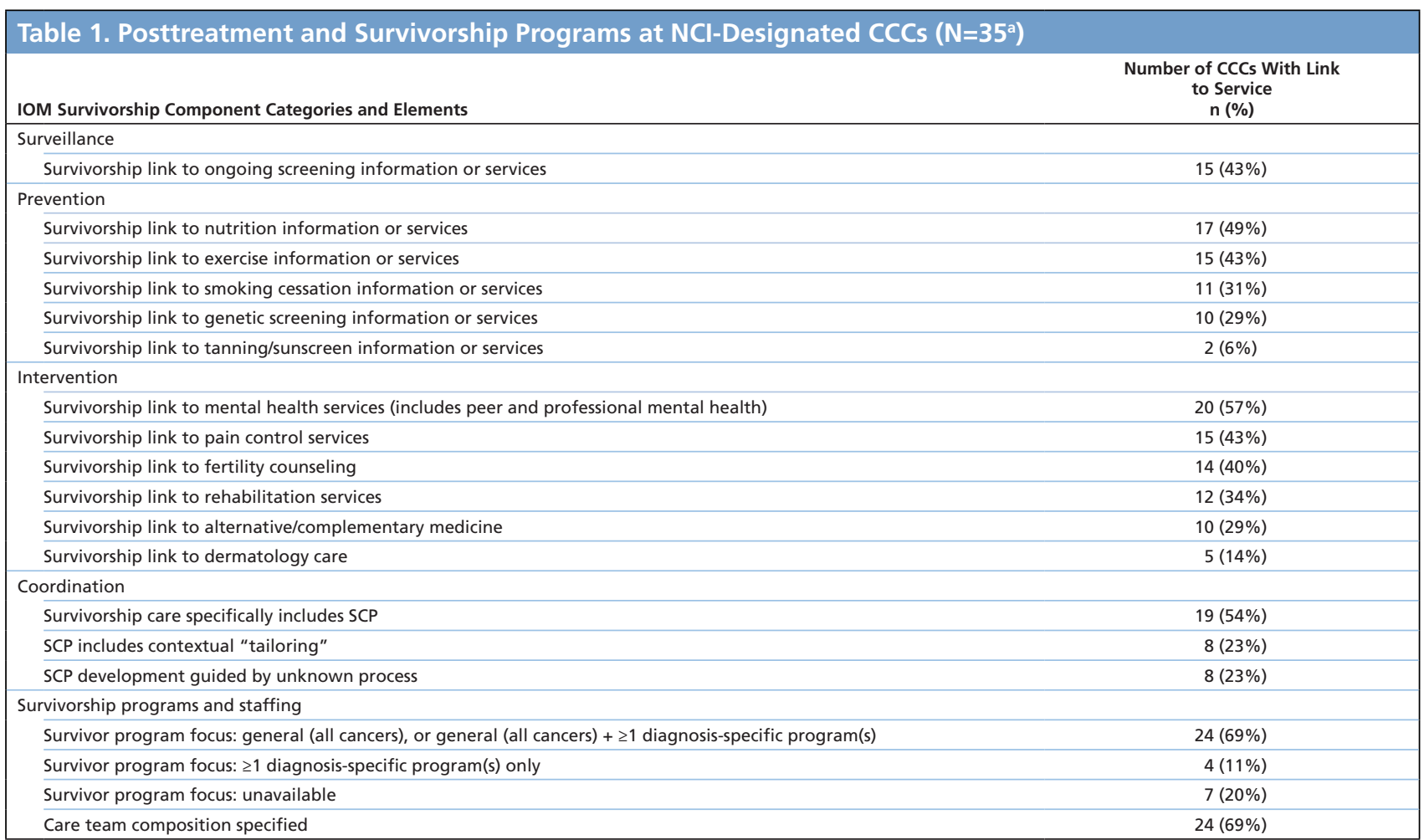

Abbreviations: CCC, Comprehensive Cancer Center; IOM, Institute of Medicine; SCP, survivorship care plan.

${ }^{a}$ Of the $47 \mathrm{NCl}$-designated CCCs assessed, 35 had information about survivorship on their websites.

mation), 9,10 no survivorship information was found. Therefore, only the 35 sites identified through the initial search were included.

For the coding process, we visited the official website of each of the 35 CCCs that returned any results in our initial search. To ensure that we did not miss any references to survivor information or care resources, we used a systematic evaluation of each site, exploring every menu item on the home page to assess content relevance to survivorship. Each coder viewed their assigned sites $\geq 2$ times, and the average time for systematically reviewing site content was approximately an hour per site. Using this technique, the coders met regularly and began to characterize strategies used for presenting survivorship information, such as offering a standalone page of content for survivors, including survivorship information on several areas of the site, or using language other than survivorship (eg, "Living Beyond Cancer") to highlight relevant content. These meetings served as a way to peer-check coding work, and to ensure no survivorship content was inadvertently missed.

The authors each coded 25 CCCs websites, with an overlap of 8 sites that both coders analyzed (32\%).
Each coder saved a static .html file with the coding data, because websites may change over time. After several iterations and discussion about coding definitions, we used simple agreement to assess intercoder reliability. For example, for the overlapping sample of sites, we counted the number of times our codes agreed and divided by the number of components coded using the standardized spreadsheet. Simple agreement showed our assessments overlapped 84\% in initial coding, which demonstrates an acceptable agreement for multiple coders. ${ }^{11}$ If the CCC served only children or focused on childhood survivors, we eliminated it from our assessment $(n=1)$. We did not differentiate between CCCs that had a general survivorship program and those that offered only diseasespecific survivorship programs (eg, a clinic for breast cancer survivors that had no general survivorship programs indicated for survivors of other diagnoses).

This assessment was limited in several ways. First, by using the search terms "survivorship" or "survivor," we may have missed services that used different terms, such as "posttreatment." Second, we were able to identify only the resources available on the CCCs' 
Rolland and Eschler

websites, which may not reflect all services available; specifically, this study relates to the findability of survivorship services, and the method cannot assess availability. Third, we may have missed other, innovative services that do not fit within the IOM components, especially areas of emphasis that may have emerged since the publication of the IOM report, such as financial toxicity, or services provided by external partners working closely with the CCC. Fourth, this assessment represents a snapshot in time. The analysis was conducted over a period of approximately 4 weeks in early 2017. Websites are updated regularly and the information may have changed since our assessment.

\section{Results}

Of the 47 CCCs, $74.5 \%(n=35)$ had discoverable survivor-specific services described on their websites. Of those 35, 8 had a survivorship link on their home page, with the remainder requiring 2 to 4 clicks to arrive at the main survivorship page. Most commonly, survivorship pages were found by following the path to patient and family services. CCCs used 2 strategies to provide survivorship information: (1) offering just one page of information and a phone number to call, or (2) offering many pages of content spread over several areas of the site, including links to external resources and non-survivor-specific services. There was very little consistency across CCCs in the services offered, the way different CCCs described survivorship care, or the role of the CCC in continuing care. The level of detail of information presented varied greatly, from a short page of general information about the patient's role in care after cancer treatment to more specific details about care available at the CCC. Although it was not always possible to discern a specific target audience for the information on each site, no site explicitly addressed materials to caregivers or clinicians (Table 1 ).

\section{Care Components}

Surveillance: Because of their high risk of recurrence or development of new cancers, survivors are advised to be vigilant about regular screening. ${ }^{3}$ However, just $43 \%(n=15)$ of the 35 CCCs had explicit information for survivors about the need to continue screening after treatment ended, and details were sparse about how or where that screening should oc- cur or about the fact that survivors might now be considered high-risk for other types of cancers.

Prevention: Preventing a new cancer or a recurrence of the primary cancer is a second major component of survivorship care. However, fewer than half $(n=17)$ of the 35 CCCs discussed nutrition or other prevention activities, 15 discussed exercise, 11 discussed smoking cessation, 10 mentioned genetic screening, and 2 discussed tanning/sunscreen use (Table 1). Again, these numbers include only the CCCs that explicitly discussed prevention services with respect to cancer survivors. Information provided on prevention topics was generally vague, and might advise survivors to "be active" or "eat a healthy diet," without any mention of available supportive services or tips to help survivors achieve these goals.

Intervention: After completing treatment, survivors may need supportive and rehabilitative services, ranging from psychosocial to physical. Despite a focus in the field of survivorship on improving the mental and emotional health of survivors, ${ }^{12}$ just 20 CCCs had survivor-specific information or services related to mental health. This was true even with our generous definition of this care component, which included everything from peer-to-peer support (eg, support groups) to professional counseling (eg, with a social worker). Again, the information available at the CCCs varied among sites, with just 15 describing services involving pain management, 14 offering some type of fertility counseling, 12 listing available rehabilitation services, 10 describing alternative or complementary medicine services, and 5 noting services or information about dermatology care in response to skin changes from cancer treatments.

Coordination: Despite the focus by the Commission on Cancer of the American College of Surgeons on providing SCPs to all cancer survivors, ${ }^{13}$ just more than half $(n=19)$ of 35 CCCs had any reference to such plans, either noting that an SCP would be created by the patient's oncologist for communicating basic information to the patient's primary care provider, or describing an SCP in greater detail and the kind of information that would be included. For example, one CCC noted that the plan would include information about treatments and drugs the patient was given, but also information about symptoms to watch for, common long-term side effects, how to 
maintain health and well-being, and employment and health insurance.

Of the 19 CCCs that referenced SCPs, just 11 described the process through which these plans would be created. Furthermore, despite evidence that culturally and ethnically tailored interventions are necessary to reduce cancer treatment outcome disparities, including survivorship care, just 8 CCCs had information about tailoring the SCP to the individual, and even fewer $(n=4)$ offered survivorship-specific information in languages other than English. ${ }^{14}$

\section{Survivorship Programs and Staffing}

CCCs that offered survivorship services did so either for all cancer survivors or survivors of specific cancers (eg, breast or prostate), or did not specify the type of program. A total of 24 CCCs offered information about a general survivorship program or both a general program and diagnosis-specific program, whereas 4 offered information only about diagnosisspecific programs; the remaining $7 \mathrm{did}$ not appear to offer survivorship programs (Table 1).

We also assessed whether the survivorship care page described the professionals offering survivorship care, because this information can give survivors clues about the types of care they can expect from the clinic. At the 24 sites (69\%) that had this information available, types of care ranged from social worker-led teams to interdisciplinary teams of doctors, advanced practice providers, and nurses.

\section{Discussion}

Our assessment found that, from the vantage point of cancer survivors and their lay caregivers, loved ones, or clinicians, limited information about survivor-specific services is available on the websites of the $47 \mathrm{NCI}$-designated CCCs. Of the 4 components of survivorship care put forth by the IOM report, only coordination and intervention were discussed by at least half of the websites we visited. A total of 11 CCCs had content in all 4 areas of the IOM guidelines, 9 had content in 3 areas, 4 had content in 2 areas, and 5 had content in only 1 area. An additional 6 CCCs mentioned survivorship but had no survivor-specific content in any of the 4 areas. Among the websites with findable survivor-specific resources, those of The University of Texas MD Anderson Cancer Center, Fred Hutchinson/
University of Washington Cancer Consortium, and Memorial Sloan Kettering Cancer Center stood out as particularly rich sources of information and can serve as models for other CCCs.

Although it is probable that general cancersupport services, such as nutrition counseling, exercise classes, and genetic screening, are available to cancer survivors at the CCCs, the lack of explicit information directed at survivors may deter them from seeking, finding, or engaging with these more general services. This potential disconnect is especially true for the CCCs that have only diagnosis-specific clinics, which may discourage survivors of other cancers from seeking help at all. Without an explicit signal that a service is right for or can accommodate the specific needs of survivors, the decision to investigate further creates extra work for survivors. This dearth of information impacts not just survivors, but also their caregivers and even clinicians unfamiliar with caring for cancer survivors.

Finally, information about survivorship care that is available is often presented in generalities, with few details to help survivors decide whether the CCC can help them with a specific aspect of their transition from active treatment to posttreatment life, or to help clinicians guide their patients. Survivors seeking information about care were urged to make an appointment to access information about services available, which might present a barrier to those struggling with side effects of their cancer treatment, such as disability or financial toxicity.

Clearly, there is an opportunity for CCCs to serve as role models for both delivery of care and provision of information by making resources about survivorship services and care offerings more findable for cancer survivors. Although this might seem like a daunting task, CCCs can start by assessing their own sites (and perhaps those of other CCCs) to locate the elements of care described in this study to understand the challenges patients and caregivers face in finding relevant information. Our findings suggest that CCCs can implement the following changes to the survivorship care content on their websites to more effectively inform survivors about the essential nature of survivorship care and its components:

- Offer an overview of survivorship care and its role in maintaining long-term health after cancer diagnosis and treatment. 
- Host information that is clearly targeted for survivors and tailored to survivorship needs, either as original content or linked to other high-quality, evidence-based information resources (eg, the American Cancer Society). This clarity reduces the work a survivor must do to decide whether a service is appropriate. CCCs can also reach out to community practices in their catchment areas to encourage these practices to share the CCC's information sources.

- Incorporate survivorship care in the information architecture of the existing website in a way that clearly expresses the CCC's commitment to supporting survivorship care as part of its mission.

- Ensure that survivorship information and content are findable given the range of information behaviors users may use to locate such information (including searching, browsing, or encountering).

\section{Conclusions}

A CCC's website provides an opportunity to communicate its values and priorities to current and potential patients, showcasing the CCC's philosophy of care. A website that has little or no information on survivorship suggests that the CCC does not prioritize these types of services or focus on the period of transition between active and posttreatment care. As the NCI increases its focus on evaluating how a CCC serves its catchment area, as with the most recent iteration of the call for proposals (PAR-17-095), CCCs have an opportunity to serve as hubs of infor- mation for their population and nonacademic medical centers. Research on how this can best be done would be a welcome addition to the conversation about catchment areas and survivorship.

Despite NCI's decades-long focus on survivorship, CCCs are still working to develop standards of care for transitioning patients from the intensity of treatment to the less structured phase of posttreatment survivorship in a way that maximizes quality of life. One way to aid this transition is to simplify the burden of work necessary to find relevant and appropriate information about survivorship services for survivors, caregivers, and clinicians. Experts in medical information-seeking, such as medical librarians, can help survivorship programs understand the types of information survivors and their caregivers are seeking and how best to organize that information.

This assessment also raises questions about what survivor-specific services should be offered by CCCs, and which should be slated for provision by primary care clinics. Especially in rural areas, where patients with cancer may travel several hours to get to their closest CCC, is it reasonable to expect survivors to access services such as nutritional counseling or pain management at the CCC? Or should the role of the CCC be primarily as an information hub for its catchment area population? As the population of survivors continues to increase, survivorship research and the CCCs must keep these questions of patient information needs and service accessibility at the forefront of the research agenda.

\section{References}

1. de Moor JS, Mariotto AB, Parry C, et al. Cancer survivors in the United States: prevalence across the survivorship trajectory and implications for care. Cancer Epidemiol Biomarkers Prev 2013;22:561-570.

2. Bluethmann SM, Mariotto AB, Rowland JH. Anticipating the "silver tsunami": prevalence trajectories and comorbidity burden among older cancer survivors in the United States. Cancer Epidemiol Biomarkers Prev 2016;25:1029-1036.

3. Institute of Medicine and National Research Council. From Cancer Patient to Cancer Survivor: Lost in Transition. Washington, DC: The National Academies Press; 2005

4. Leppin A, Montori V, Gionfriddo M. Minimally disruptive medicine: a pragmatically comprehensive model for delivering care to patients with multiple chronic conditions. Healthcare (Basel) 2015;3:50-63.

5. Eschler J, Meas PL, Lozano P, et al. Integrating the patient portal into the health management work ecosystem: user acceptance of a novel prototype. AMIA Annu Symp Proc 2016;2016:541-550.

6. Klasnja P, Hartzler AC, Unruh KT, Pratt W. Blowing in the wind: unanchored patient information work during cancer care. Proc SIGCHI Conf Hum Factor Comput Syst 2010;2010:193-202.

7. Fox F, Duggan M. Health Online 2013. Pew Research Center: Internet \& Technology. Available at: http://www.pewinternet.org/2013/01/15/healthonline-2013/. Accessed June 13, 2018.

8. Finney Rutten LJ, Agunwamba AA, Wilson P, et al. Cancer-related in formation seeking among cancer survivors: trends over a decade (20032013). J Cancer Educ 2016;31:348-357.

9. Bates MJ. The design of browsing and berrypicking techniques for the online search interface. Online Review 1989;13:407-424.

10. Case DO. Looking for Information: A Survey of Research on Information Seeking, Needs, and Behavior. San Diego, CA: Academic Press; 2002.

11. Miles MB, Huberman AM, Saldana J. Qualitative Data Analysis: A Methods Sourcebook, 3rd ed. Thousand Oaks, CA: SAGE Publications, Inc.; 2014.

12. Carlson LE, Waller A, Mitchell AJ. Screening for distress and unmet needs in patients with cancer: review and recommendations. J Clin Oncol 2012;30:1160-1177.

13. Cancer Program Standards: Ensuring Patient-Centered Care. 2016 Edition. Available at: https://www.facs.org/ /media/files/quality\%20programs/ cancer/coc/2016\%20coc\%20standards $\% 20$ manual_interactive $\% 20$ pdf ashx. Accessed June 13, 2018

14. American Cancer Society. Cancer Facts \& Figures 2017. Atlanta, GA American Cancer Society; 2017. 